\title{
Synthesis and anti-myocarditis activity in a multifunctional lanthanide microporous metal-organic framework with 1D helical chain building units
}

\author{
Chenglv Hong ${ }^{1}$, Xinlang Zhou ${ }^{2}$, Weijian Huang ${ }^{1}$, Peiren Shan ${ }^{1}$ and Fengquan Dong ${ }^{3}$ \\ ${ }^{1}$ Department of Cardiology, The First Affiliated Hospital of Wenzhou Medical University, Wenzhou, Zhejiang, China \\ ${ }^{2}$ Department of Cardiology, Wenzhou City Hospital of Traditional Chinese Medicine and Western Medicine Combined, \\ Wenzhou, Zhejiang, China \\ ${ }^{3}$ Department of Cardiology, Shenzhen University General Hospital, Shenzhen, Guangdong, China
}

\begin{abstract}
A new microporous lanthanide metal-organic framework, $\left\{\left[\mathrm{Yb}(\mathrm{BTB})\left(\mathrm{H}_{2} \mathrm{O}\right)(\mathrm{DEF})_{2}\right\}_{\mathrm{n}}(1, \mathrm{DEF}=N, N\right.$-Diethylformamide), with 1D nano-sized channels has been constructed by bridging helical chain secondary building units with 1,3,5-benzenetrisbenzoic acid $\left(\mathrm{H}_{3} \mathrm{BTB}\right)$ ligand. Structural characterization suggests that this complex crystallizes in the hexagonal space group P6 22 and possesses 1D triangular channels with coordinated water molecules pointing to the channel center. In addition, anti-myocarditis properties of compound $\mathbf{1}$ were evaluated in vivo. The results showed that compound $\mathbf{1}$ can improve hemodynamic parameters of, and it may be a good therapeutic option for heart failure in the future.
\end{abstract}

Key words: Metal-organic framework; Anti-myocarditis; In vivo

\section{Introduction}

Myocarditis, also known as inflammatory cardiomyopathy, is the inflammation of the heart muscle. Symptoms can include shortness of breath, chest pain, decreased ability to exercise, and an irregular heartbeat $(1,2)$. The duration of problems can vary from hours to months. Complications may include heart failure due to dilated cardiomyopathy or cardiac arrest $(3,4)$.

Metal-organic frameworks (MOFs) that are constructed by coordination of metal centers with multiorganic connectors represent an emerging class of inorganic-organic hybrid crystalline materials $(5,6)$. Their structural tenability, well-defined single crystal architectures, functionalized pore environment and modifiable building blocks make them useful in many potential applications including biological activity, catalysis, and luminescent sensing materials (7-9). The organic ligand plays an important role in the construction of porous MOFs because it not only guides the formation of the secondary building units, but also determines the pore shapes and pore surroundings of the obtained products $(10,11)$. MOFs prepared with ligands of high symmetry have been well studied because of synthetic and crystallographic considerations. As the elongated ligand of $\mathrm{H}_{3} \mathrm{BTC}$, 1,3,5-benzenetrisbenzoic acid $\left(\mathrm{H}_{3} \mathrm{BTB}\right.$, Figure 1) has been widely used in the construction of porous MOFs $(12,13)$. However, compared with the transition metal-BTB frameworks reported, the lanthanon metalBTB frameworks are less studied (14).

Here, we present the synthesis and the structural analysis of a highly porous $\mathrm{Yb}$-organic network $\left\{\mathrm{Yb}(\mathrm{BTB})\left(\mathrm{H}_{2} \mathrm{O}\right)\right]$ $\left.(\mathrm{DEF})_{2}\right\}_{\mathrm{n}}(\mathbf{1}, \mathrm{DEF}=N, \mathrm{~N}$-diethylformamide). This MOF is composed of novel $1 \mathrm{D}$ helical chain building units and $\mathrm{BTB}^{3-}$ ligand, which represents the first example of Ln-MOFs based on 1D helical chain building units. In addition, in vivo anti-myocarditis activity of compound 1 was investigated.

\section{Material and Methods}

\section{Apparatus and materials}

All the starting materials and reagents used in this work were obtained commercially and used without further purification. Element analyses $(\mathrm{C}, \mathrm{H}$, and $\mathrm{N})$ were determined with an elemental Vairo EL III analyzer (Bruker, Germany). Powder X-ray diffraction data were collected using PANalytical X'Pert Pro powder diffractometer (Bruker) with $\mathrm{Cu}-\mathrm{K} \alpha$ radiation and $5^{\circ} \leqslant 2 \theta \leqslant 50^{\circ}$. Thermogravimetric experiments were performed using a TGA/NETZSCH STA449C instrument heated from 30 to $800^{\circ} \mathrm{C}$ (heating rate of $10^{\circ} \mathrm{C} / \mathrm{min}$, nitrogen stream; Bruker). Single crystal 
X-ray diffraction was carried out by an Oxford Xcalibur E diffractometer (Bruker).

\section{Synthesis and characterization of $\left\{\left[\mathrm{Yb}(\mathrm{BTB})\left(\mathrm{H}_{2} \mathrm{O}\right)\right]\right.$ $\left.(\mathrm{DEF})_{2}\right\}_{\mathrm{n}}(1)$}

A mixture of $\mathrm{Yb}\left(\mathrm{NO}_{3}\right)_{2} \cdot 6 \mathrm{H}_{2} \mathrm{O}(0.1 \mathrm{mmol}, 0.031 \mathrm{~g})$ and $\mathrm{H}_{3} \mathrm{BTB}$ (35 mg, $0.062 \mathrm{mmol}$ ) was added to a solution of DEF $(4 \mathrm{~mL})$ and $\mathrm{H}_{2} \mathrm{O}(1 \mathrm{~mL})$. The mixture was sealed in a Pyrex tube, and heated at $140^{\circ} \mathrm{C}$ for 3 days. After cooling to room temperature, the colorless polyhedral-shaped<smiles>O=C(O)c1ccc(-c2cc(-c3ccc(C(=O)O)cc3)cc(-c3ccc(C(=O)O)cc3)c2)cc1</smiles>

Figure 1. Schematic representation of the $\mathrm{H}_{3} \mathrm{BTB}$ ligand used in this research crystals formed were filtered, washed with DEF, and then dried in air. Analytical data for compound $1\left(\mathrm{C}_{37} \mathrm{H}_{39} \mathrm{~N}_{2}\right.$ $\mathrm{O}_{9} \mathrm{Yb}$ ): C, 53.23; H, 4.44; N, 3.29\%. Calculated: C, 53.62; $\mathrm{H}, 4.74 ; \mathrm{N}, 3.38 \%$.

\section{Crystal structure determination}

Suitable single crystal of compound 1 was carefully selected under optical microscope and glued on thin glass fibers. The intensity data of 1 was collected on an Oxford Xcalibur E diffractometer. The empirical absorption corrections were applied to the data using the SADABS system. This structure was solved by direct method and refined by full-matrix least-squares method on $F^{2}$ using the SHELXS-97 program (15). All non-hydrogen atoms of 1 were refined anistropically, and all the hydrogen atoms attached to carbon atoms were fixed at their ideal positions. Pertinent crystal data and structural refinement results for compound 1 are summarized in Table 1.

\section{In vivo anti-myocarditis activity}

C57BL6/j mice were involved in our experiment. A total of 48 eight-week-old male mice were divided into four groups: control + PBS (G1, n=12), control +1 (G2, n=12), CVB3 + PBS (G3, n=12), CVB3+1 (G4, n=12). G3 and G4 were infected by intraperitoneal (ip) injection of $1 \times 10^{5}$

Table 1. Crystal data and structure refinements for compound 1.

\begin{tabular}{ll}
\hline Formula weight & 624.43 \\
Temperature/K & $293(2)$ \\
Crystal system & hexagonal \\
Space group & $\mathrm{P} 6_{1} 22$ \\
$\mathrm{a} / \AA$ & $18.0081(15)$ \\
$\mathrm{b} / \AA$ & $18.0081(15)$ \\
$\mathrm{c} / \AA$ & $21.8141(13)$ \\
$\alpha / /^{\circ}$ & 90 \\
$\beta / /^{\circ}$ & 90 \\
$\gamma /{ }^{\circ}$ & 120 \\
Volume/ $\AA^{3}$ & $6126.4(11)$ \\
$Z$ & 6 \\
$\rho_{\text {calc }} / \mathrm{cm}^{3}$ & 1.016 \\
$\mu / \mathrm{mm}^{-1}$ & 2.316 \\
Radiation & MoK $\alpha(\lambda=0.71073)$ \\
$2 \Theta$ range for data collection ${ }^{\circ}$ & 6.422 to 52.726 \\
Reflections collected & 16173 \\
Independent reflections & $4186\left[\mathrm{R}_{\text {int }}=0.0485, \mathrm{R}_{\text {sigma }}=0.0462\right]$ \\
Data/restraints/parameters & $4186 / 111 / 162$ \\
Goodness-of-fit on $\mathrm{F}^{2}$ & 1.018 \\
Final $\mathrm{R}$ indexes $[\mathrm{l}>=2 \sigma(\mathrm{I})]$ & $\mathrm{R}_{1}=0.0316, \omega \mathrm{R}_{2}=0.0684$ \\
Final $\mathrm{R}$ indexes [all data] & $\mathrm{R}_{1}=0.0440, \omega \mathrm{R}_{2}=0.0734$ \\
Largest diff. peak/hole $/ \mathrm{e} \AA \AA^{-3}$ & $0.42 /-0.78$ \\
Flack parameter & $-0.026(10)$ \\
CCDC & 1573543 \\
\hline &
\end{tabular}

CCDC: Cambridge Crystallographic Data Centre. 
A

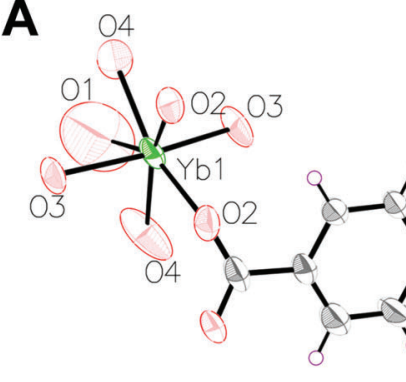<smiles>C1CCCCC1</smiles><smiles>C1CCCCC1</smiles>

\section{C}

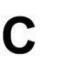

B
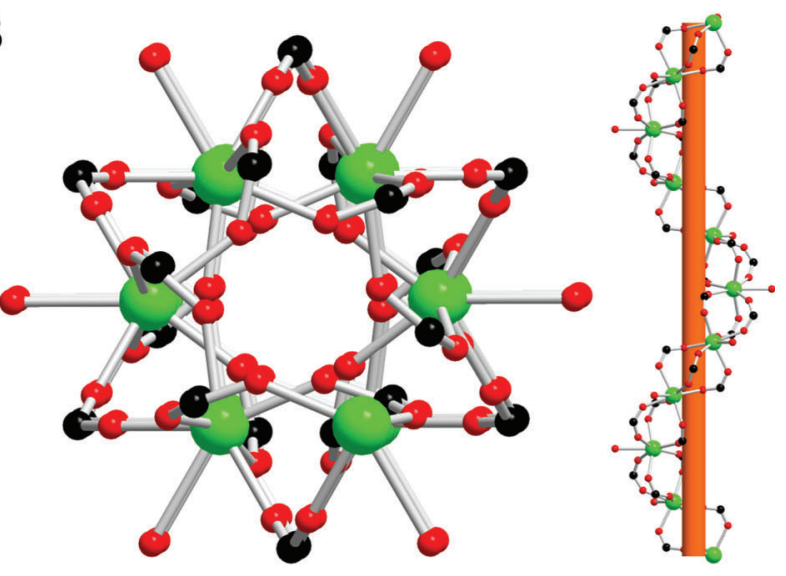

D
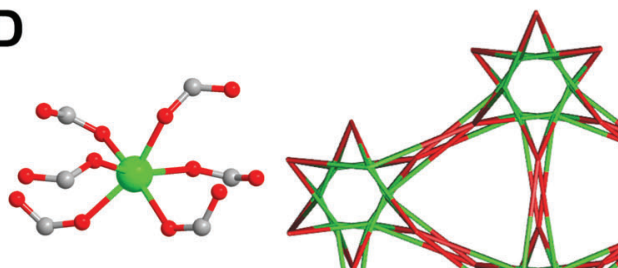

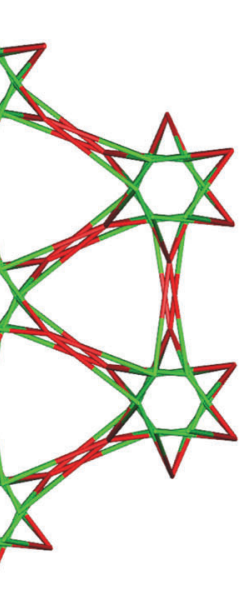

Figure 2. $A$, view of the asymmetric unit in compound 1 with $30 \%$ thermal ellipsoid level; $B$, view of $1 D$ helical chain building units in 1 ; $C$, view of the $1 \mathrm{D}$ triangular channels in $1 ; D,(6,6)$-connected topology for 1.

plaque forming units (pfu) Coxsackie virus B3 (CVB3) per mouse, while $\mathrm{G} 1$ and $\mathrm{G} 2$ received ip injection of phosphate-buffered saline (PBS) on the same day. Compound 1 was orally applied at $50 \mathrm{mg} / \mathrm{kg}$ on the next day of infection (G2 and G4), while G1 and G3 were orally administrated the same dose of PBS. Animals were housed with a normal diet, $12 \mathrm{~h}$ light/dark cycle, $30-70 \%$ humidity, and $20-25^{\circ} \mathrm{C}$. All mice were sacrificed on day 7 post-CVB3 infection. We used a conductance catheter (DDS-307, Chang-Ai, China) to collect hemodynamic data (pressure and volume) before sacrificing the animals.

Statistical analysis was performed using Prism 6 (Bruker). One-way analysis of variance (ANOVA) was used for statistical analysis of the data with correction for multiple comparisons via the Tukey's range test. Data are reported as means $\pm S D$. Differences were regarded to be significant if the two-sided P-value was lower than 0.05 .

\section{Results and Discussion}

\section{Molecular structure}

The solvothermal reaction of $\mathrm{Yb}\left(\mathrm{NO}_{3}\right)_{3} \cdot 6 \mathrm{H}_{2} \mathrm{O}$ and $\mathrm{H}_{3}$ BTB in a mixed solvent of DEF and $\mathrm{H}_{2} \mathrm{O}$ provided complex 1 as colorless crystals. Single-crystal X-ray diffraction reveals that 1 crystallizes in a highly symmetric and chiral hexagonal space group $P 6_{1} 22$ and the 3-D coordination network is constructed through the connection of infinite 1-D helical chain building units and the $\mathrm{BTB}^{3-}$ ligands. The asymmetric coordination unit consists of one $\mathrm{Yb}$ ion situated on a symmetry site with one half occupancy, half BTB $^{3-}$ ligand and one coordinated water molecule. As shown in Figure $2 A$, the $Y b(I I I)$ ion is sevencoordinated by six carboxylic acid $O$ atoms from six different $\mathrm{BTB}^{3-}$ ligands and one coordinated water molecule, resulting in a pentagonal bipyramid geometry. The $\mathrm{Yb}-\mathrm{O}$ bond distances are in the range of 2.212 (3) to 2.609 (5) $\AA$. 

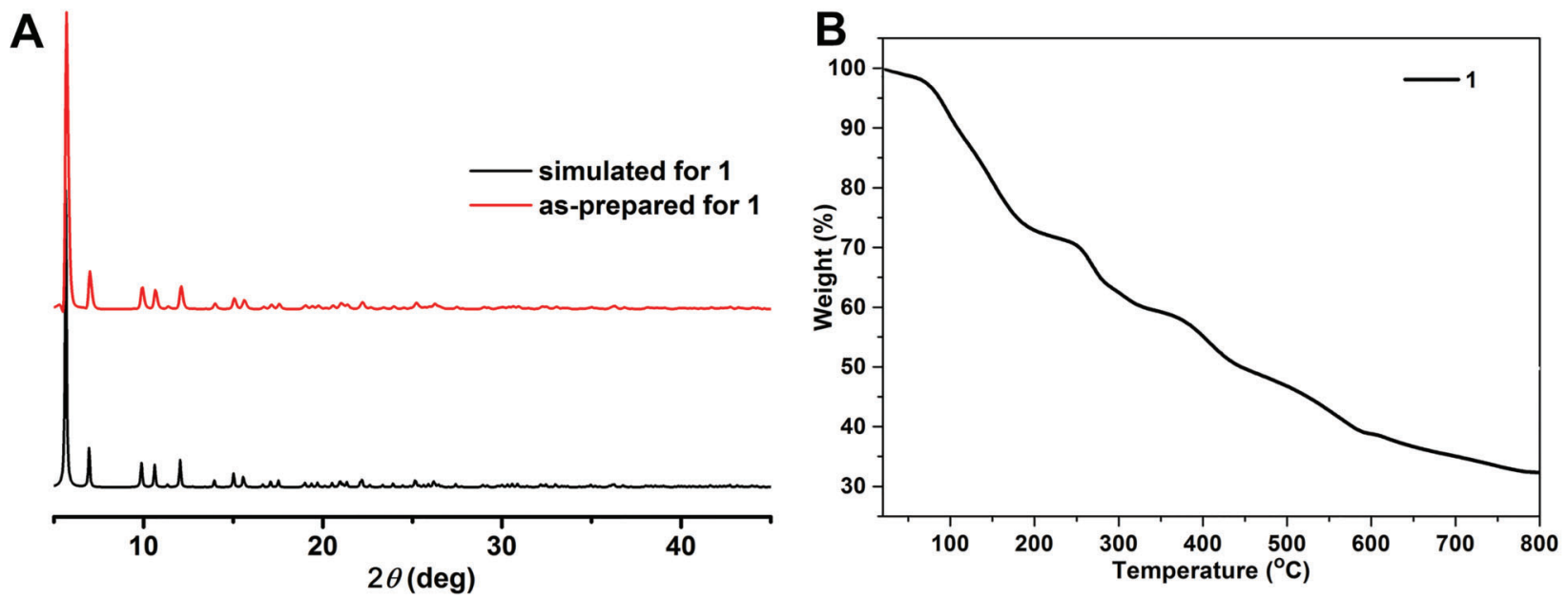

Figure 3. A, powder X-ray diffraction analysis patterns for compound 1. B, TGA curve for 1.

Table 2. Body weight of mice at day 0 and day 7 .

\begin{tabular}{lclll}
\hline & Control + PBS & Control +1 & CVB3 +PBS & \multicolumn{1}{c}{ CVB3+1 } \\
\hline Day 0 BW $(\mathrm{g})$ & $24.83 \pm 0.66$ & $24.88 \pm 0.69$ & $24.88 \pm 0.64$ & $24.79 \pm 0.59$ \\
Day 7 BW $(\mathrm{g})$ & $26.65 \pm 0.49$ & $26.37 \pm 0.74$ & $20.41 \pm 0.73^{\star \#}$ & $22.41 \pm 1.15^{\star \#+}$ \\
\hline
\end{tabular}

Data are reported as means \pm SD. BW: body weight. CVB3: Coxsackie virus B3; PBS: phosphate buffered saline. ${ }^{*} \mathrm{P}<0.01$ vs control + PBS, ${ }^{\#} \mathrm{P}<0.01$ vs control $+1,{ }^{+} \mathrm{P}<0.01$ vs $\mathrm{CVB} 3+\mathrm{PBS}$ (one-way ANOVA).

Each $\mathrm{Yb}$ atom is connected with the neighboring ones though three carboxylic groups. Such a connection mode leads to the formation of a 1D right-handed chain along a $6_{1}$ axis, which represent a rare case of Ln-based helical chain building units according to the Cambridge Crystallographic Data Centre database (Figure 2B). The pitch of the helical chain is 21.832 (3) A. Furthermore, such 1D helical chain building units are further linked by BTB ligand through its three carboxylate groups to afford a 3D noninterpenetrating framework with 1D triangular channels with coordinated water molecule pointing to the channel center (Figure 2C). Based on the crystallographic data and considering the van der Waals radii of atoms, the pore size for the triangular channel is $5.4 \AA$. To understand the network of 1 more clearly, we use the software TOPOS to simplify its framework. Each $\mathrm{Yb}$ (III) ion is connected with six $O$ atoms from six different BTB $^{-}$ligands and each $\mathrm{BTB}^{-}$ligand binds with six different $\mathrm{Yb}(\mathrm{III})$ ions. Thus, both of the $\mathrm{Yb}(\mathrm{III})$ ions and the $\mathrm{BTB}^{-}$ligand could be viewed as 6 -connected nodes. In this case, the whole framework of 1 can be simplified to a 2-nodal $(6,6)$-connected network with the point symbol of $\left(4^{10} .6^{5}\right)\left(4^{7} .6^{8}\right)$, which has not been observed in MOF chemistry (Figure 2D). The effective free volume of 1 without guest water molecules is $58.3 \%$ of the crystal volume $\left(3569 \AA^{3}\right.$ of the $6126 \AA^{3}$ unit cell volume), calculated with PLATON software.

\section{Powder X-ray diffraction analysis (PXRD) and thermal analysis}

PXRD experiment was carried out to verify the phase purity of the as-synthesized samples. As shown in Figure 3A, the diffraction peak of compound 1 is in good agreement with that of the simulated one based on the single crystal diffraction data, indicating the pure phase of the obtained samples. From the thermogravimetric curve of compound 1, we found that the first weight loss of $27.1 \%$ occurs from 25 to $210^{\circ} \mathrm{C}$, which corresponds to the release of one coordinated water molecule and two lattice DEF molecules (Calcd: $26.5 \%$ ). Then, the dissolved sample was stable up to $230^{\circ} \mathrm{C}$, after which the framework began to collapse (Figure 3B).

\section{In vivo anti-myocarditis activity}

To evaluate the impact of compound 1 in CVB3induced myocarditis, body weight, heart rate (HR), maximum left ventricle pressure (Pmax), maximum left ventricle pressure rise rate (dP/dtmax), and ejection fraction $(E F)$ were analyzed in the present study. There was a significant difference in body weight between G3 and G1. Furthermore, there was a significant difference between G4 and G3 (Table 2). In comparison to G1 and $\mathrm{G} 2$, there was a sharp decrease in $\mathrm{G} 3$ animals in $H R$, Pmax, dP/dtmax and EF. Oppositely, G4 had a downward 
Table 3. Hemodynamic data of mice.

\begin{tabular}{lcccc}
\hline & Control +PBS & Control +1 & CVB3 +PBS & CVB3+1 \\
\hline HR $(\mathrm{bpm})$ & $531.48 \pm 40.32$ & $529.98 \pm 39.46$ & $386.77 \pm 124.59^{\star \#}$ & $475.79 \pm 79.13^{+}$ \\
Pmax $(\mathrm{mmHg})$ & $113.96 \pm 21.64$ & $109.66 \pm 12.91$ & $90.25 \pm 22.37^{\star \#}$ & $105.34 \pm 11.43$ \\
dP/dtmax $(\mathrm{mmHg} / \mathrm{s})$ & $9742.27 \pm 1766.32$ & $9445.64 \pm 1920.23$ & $6108.48 \pm 2592.93^{\star \#}$ & $8000.47 \pm 1378.99$ \\
EF $(\%)$ & $78.60 \pm 2.65$ & $77.76 \pm 5.20$ & $69.66 \pm 7.74^{\star \#}$ & $73.64 \pm 4.59$ \\
\hline
\end{tabular}

Data are reported as means $\pm \mathrm{SD}$. HR: heart rate; Pmax: maximum left ventricle pressure; $\mathrm{dP} / \mathrm{dtmax}$ : maximum rate of rise of left ventricle pressure; EF: ejection fraction; CVB3: coxsackie virus B3; PBS: phosphate buffered saline. ${ }^{*} \mathrm{P}<0.05$ vs control $+\mathrm{PBS}$, ${ }^{\#} \mathrm{P}<0.05$ vs control + compound 1, ${ }^{+} \mathrm{P}<0.01$ vs CVB3 + PBS (one-way ANOVA).

trend that compared to $\mathrm{G} 1$ and $\mathrm{G} 2$, but there was no significant difference between them (Table 3).

As known, human myocarditis can result in chest discomfort, palpitation, shortness of breath, dizziness, decreased activity, and poor appetite. CVB3-mice are a good myocarditis model that we can easily see reduced activity, and get body weight data through weighing; the decreased appetite indicates that the myocarditis model works. From our in vivo experiment, we found that the CVB3 group significantly lost body weight, but it seemed to reverse after application of compound 1. The CVB3 group had a significant decrease of HR, Pmax, dP/dtmax, and EF, which are essential factors of heart failure, especially

\section{References}

1. Ghatnur SM, Parvatam G, Balaraman M. Culture conditions for production of biomass, adenosine, and cordycepin from cordyceps sinensis CS1197: optimization by desirability function method. Pharmacogn Mag 2015; 11 (Suppl 3): S448S456, doi: 10.4103/0973-1296.168946.

2. Root-Bernstein R, Fairweather D. Unresolved issues in theories of autoimmune disease using myocarditis as a framework. J Theor Biol 2015; 375: 101-123, doi: 10.1016/ j.jtbi.2014.11.022.

3. Li-Sha G, Yi-He C, Na-Dan Z, Teng Z, Yue-Chun L. Effects of carvedilol treatment on cardiac cAMP response element binding protein expression and phosphorylation in acute coxsackievirus B3-induced myocarditis. BMC Cardiovasc Disord 2013; 13: 100, doi: 10.1186/1471-2261-13-100.

4. Massilamany C, Gangaplara A, Reddy J. Intricacies of cardiac damage in coxsackievirus B3 infection: Implications for therapy. Int J Cardiol 2014; 177: 330-339, doi: 10.1016/ j.ijcard.2014.09.136.

5. Krejci J, Mlejnek D, Sochorova D, Nemec P. Inflammatory cardiomyopathy: a current view on the pathophysiology, diagnosis, and treatment. Biomed Res Int 2016; 2016: 4087632, doi: 10.1155/2016/4087632.

6. Li-Sha G, Jing-Lin Z, Guang-Yi C, Li L, De-Pu Z, Yue-Chun $L$. Dose-dependent protective effect of nicotine in a murine model of viral myocarditis induced by coxsackievirus B3. Sci Rep 2015; 5: 15895, doi: 10.1038/srep15895.

7. Nyland JF, Fairweather D, Shirley DL, Davis SE, Rose NR, Silbergeld EK. Low-dose inorganic mercury increases severity and frequency of chronic coxsackievirus-induced autoimmune systolic heart failure. In our investigation, compound 1 was effective in hemodynamics, indicating it could be a candidate for anti-myocarditis therapy.

In conclusion, we demonstrated the successful construction of a novel $\mathrm{Yb}$-based MOF with 1D helical chain building units built up from 1,3,5- $\mathrm{H}_{3}$ BTB ligand. Structural characterization suggests that this complex crystallizes in the hexagonal space group $P 6_{1} 22$ and possesses 1D triangular channels with coordinated water molecules pointing to the channel center. In addition, the results showed that compound $\mathbf{1}$ can improve hemodynamic parameters, and may be a good therapeutic compound for heart failure in the future. myocarditis in mice. Toxicol Sci 2012; 125: 134-143, doi: 10.1093/toxsci/kfr264.

8. Junghans $\mathrm{U}$, Kobalz $\mathrm{M}$, Erhart $\mathrm{O}$, Preißler $\mathrm{H}$, Lincke $\mathrm{J}$, Möllmer J, Krautscheid H, Gläser R. A Series of robust copper-based triazolyl isophthalate MOFs: impact of linker functionalization on gas sorption and catalytic activity. Materials 2017; 10: 338, doi: 10.3390/ma10040338.

9. Haydar MAL, Abid HR, Sunderland B, Wang S. Metal organic frameworks as a drug delivery system for flurbiprofen. Drug Des Devel Ther 2017; 11: 2685-2695, doi: 10.2147/DDDT.S145716.

10. Han Q, Qi B, Ren W, He C, Niu J, Duan C. Polyoxometalate-based homochiral metal-organic frameworks for tandem asymmetric transformation of cyclic carbonates from olefins. Nat Commun 2015; 6: 10007, doi: 10.1038/ ncomms10007.

11. Kim JY, Jin M, Lee KJ, Cheon JY, Joo SH, Kim JM, et al. In situ-generated metal oxide catalyst during $\mathrm{CO}$ oxidation reaction transformed from redox-active metal-organic framework-supported palladium nanoparticles. Nanoscale Res Lett 2012; 7: 461, doi: 10.1186/1556-276X-7-461.

12. Wang Z, Wang J, Li M, Sun K, Liu C. Three-dimensional printed acrylonitrile butadiene styrene framework coated with Cu-BTC metal-organic frameworks for the removal of methylene blue. Sci Rep 2014; 4: 5939, doi: 10.1038/ srep05939.

13. Mitra J, Guerrero EN, Hegde PM, Wang H, Boldogh I, Rao $\mathrm{KS}$, et al. New perspectives on oxidized genome damage and repair inhibition by pro-oxidant metals in neurological 
diseases. Biomolecules 2014; 4: 678-703, doi: 10.3390/ biom4030678.

14. Zhu Y, Zhu M, Xia L, Wu Y, Hua H, Xie J. Lanthanide metalorganic frameworks with six-coordinated $\operatorname{In}($ iii) ions and free functional organic sites for adsorptions and extensive catalytic activities. Sci Rep 2016; 6: 29728, doi: 10.1038/ srep29728.

15. Sheldrick GM. SHELXL-97, program for crystal structure solution and refinement. University of Göttingen: Göttingen, Germany; 1997. 